\title{
Les « portraits de portrait » de Jim Campbell : ambiguïté et traitement de l'information
}

\section{Isabelle Masse}

Volume 39, numéro 1, 2014

URI : https://id.erudit.org/iderudit/1026202ar

DOI : https://doi.org/10.7202/1026202ar

Aller au sommaire du numéro

\section{Éditeur(s)}

UAAC-AAUC (University Art Association of Canada | Association d'art des universités du Canada)

\section{ISSN}

0315-9906 (imprimé)

1918-4778 (numérique)

Découvrir la revue

Citer cet article

Masse, I. (2014). Les " portraits de portrait » de Jim Campbell : ambiguïté et traitement de l'information. RACAR : Revue d'art canadienne / Canadian Art Review, 39(1), 52-65. https://doi.org/10.7202/1026202ar

\section{Résumé de l'article}

The ability or inability to perceive facial features and expressions has a direct impact on communication. In this sense, the clarity or elusiveness of the image in the experience of portraiture should influence spectatorship. It is from this perspective that American artist Jim Campbell uses scientific references to explore the effects of visual ambiguity in electronic portraits belonging to his series "Ambiguous Icons." This article focuses on two of these portraits, namely a matching pair in which the medium significantly obfuscates the representation: Portrait of a Portrait of Harry Nyquist and Portrait of a Portrait of Claude Shannon (2000). The sitters are two scientists whose work laid the foundation for information theory, a mathematical model of communication originally created for telecommunications, but whose applications later extended to cognitive science. I examine how these artworks, by material and formal means, can be understood as artistic analogies of information theory and then argue that through this theoretical reference they evoke a traditional model of human cognition. Lastly, I discuss the strategies adopted to elicit the viewer's response from two perspectives: the perceptual experience and, more broadly, the aesthetic experience, respectively analyzed through the artist's reinterpretation of a 1973 experiment on facial recognition and through a 1960s transposition of information theory to the arts.
Tous droits réservés (C) UAAC-AAUC (University Art Association of Canada | Association d'art des universités du Canada), 2014
Ce document est protégé par la loi sur le droit d'auteur. L'utilisation des services d'Érudit (y compris la reproduction) est assujettie à sa politique d'utilisation que vous pouvez consulter en ligne.

https://apropos.erudit.org/fr/usagers/politique-dutilisation/ 


\title{
Les « portraits de portrait » de Jim Campbell : ambiguité et traitement de l'information
}

\author{
Isabelle Masse, McGill University
}

\begin{abstract}
The ability or inability to perceive facial features and expressions has a direct impact on communication. In this sense, the clarity or elusiveness of the image in the experience of portraiture should influence spectatorship. It is from this perspective that American artist Jim Campbell uses scientific references to explore the effects of visual ambiguity in electronic portraits belonging to his series "Ambiguous Icons." This article focuses on two of these portraits, namely a matching pair in which the medium significantly obfuscates the representation: Portrait of a Portrait of Harry Nyquist and Portrait of a Portrait of Claude Shannon (2000). The sitters are two scientists whose work laid the foundation for information theory, a mathematical model of communication originally created for telecommunications, but whose applications later extended to cognitive science. I examine how these artworks, by material and formal means, can be understood as artistic analogies of information theory and then argue that through this theoretical reference they evoke a traditional model of human cognition. Lastly, I discuss the strategies adopted to elicit the viewer's response from two perspectives: the perceptual experience and, more broadly, the aesthetic experience, respectively analyzed through the artist's reinterpretation of a 1973 experiment on facial recognition and through a 1960s transposition of information theory to the arts.
\end{abstract}

L L'information qu'il transmet, à travers les traits et les expressions faciales, détermine l'interprétation de celui qui regarde et, du même coup, son comportement social. En ce sens, la capacité ou l'incapacité à percevoir les physionomies influe directement sur les rapports communicationnels. Aussi, dans l'expérience esthétique du portrait, l'insaisissabilité de l'image véhiculerait-elle sans doute une charge d'effet ayant, à des degrés divers, une incidence sur la réaction du spectateur. C'est dans cette optique que des portraits appartenant à la série "Ambiguous Icons" de l'artiste américain Jim Campbell (né en 1956) explorent, à l'aide de références scientifiques, l'impact de l'ambiguïté visuelle sur la réponse spectatorielle. Le présent article se penche sur deux de ces portraits, soit deux pendants dans lesquels le travail du médium altère la perceptibilité des visages : Portrait of a Portrait of Harry Nyquist et Portrait of a Portrait of Claude Shannon (2000) (fig. 1 et 2). Les deux œuvres s'inscrivent dans le cadre d'un projet de recherche artistique ayant bénéficié de l'appui financier de la Fondation Daniel Langlois pour l'art, la science et la technologie ${ }^{1}$. Intitulé Explorations of Meaning in Quantized Information, le projet a trouvé son aboutissement avec un corpus d'œuvres expérimentales qui prennent la forme de dispositifs vidéographiques conçus sur mesure à l'aide de matériel électronique. Dans la réalisation de ce travail, Jim Campbell, un diplômé du MIT, tire parti de ses compétences en mathématiques et en génie électrique ${ }^{2}$ : il conçoit ses propres logiciels, expérimente avec le médium électronique et fabrique, dans la foulée, des dispositifs uniques dont les possibilités excèdent celles de la technologie couramment utilisée. La souplesse avec laquelle l'artiste façonne son médium fait plus que répondre adéquatement à des intentions conceptuelles : elle permet de moduler avec précision les déterminants sensibles de l'œuvre d'art, soit d'organiser les stimuli visuels afin d'orienter, autant que faire se peut, les paramètres d'une expérience perceptuelle et esthétique.

Si la notion d'identité demeure la pierre angulaire d'une majorité d'analyses sur le portrait, elle ne constitue pas, dans les œuvres de Jim Campbell, un véritable enjeu de la représentation. En effet, le travail du médium entrave la perceptibilité des visages au point où l'ambiguïté qui en découle pose un défi à l'attribution de significations claires. L'opacification de l'image tend ainsi à démentir l'habituelle fonction identitaire du portrait, une fonction de construction ou de déconstruction dans laquelle se condense, le plus souvent, la signification de l'image. Pour tout dire, les œuvres ne sont pas strictement référentielles : elles ne désignent pas que les visages de deux scientifiques. Il en résulte que l'expérience spectatorielle diffère de celle qui, dans l'historiographie, est généralement associée au portrait. En effet, les études sur le genre, qui mettent sans cesse l'accent sur le caractère référentiel de l'image, assimilent l'expérience esthétique à une réaction suscitée au contact de la figure représentée. Comme le souligne l'historien de l'art Richard Brilliant : "The agenda of such studies reflects once again the traditional emphasis on the person portrayed as the centre of empathetic and aesthetic response "3. À l'inverse, notre démarche s'attachera moins à considérer une signification ou une réaction données par le référent que d'explorer la manière dont le médium et les références scientifiques inscrites dans ce médium produisent sens et expérience.

Ainsi, l'article, divisé en quatre parties, examinera d'abord comment les portraits de Campbell proposent, par leur articulation médiale, un équivalent artistique d'une théorie scientifique à laquelle ont contribué les modèles représentés. Il soutiendra ensuite qu’à travers cette référence théorique les œuvres évoquent un modèle traditionnel de cognition humaine. Enfin, il s'intéressera à la réponse spectatorielle sous deux perspectives, soit celle de l'expérience perceptuelle et, plus largement, celle de l'expérience esthétique. La première sera analysée à travers la réinterprétation par l'artiste d'une expérimentation scientifique, datant de 1973, sur la reconnaissance faciale et le traitement 


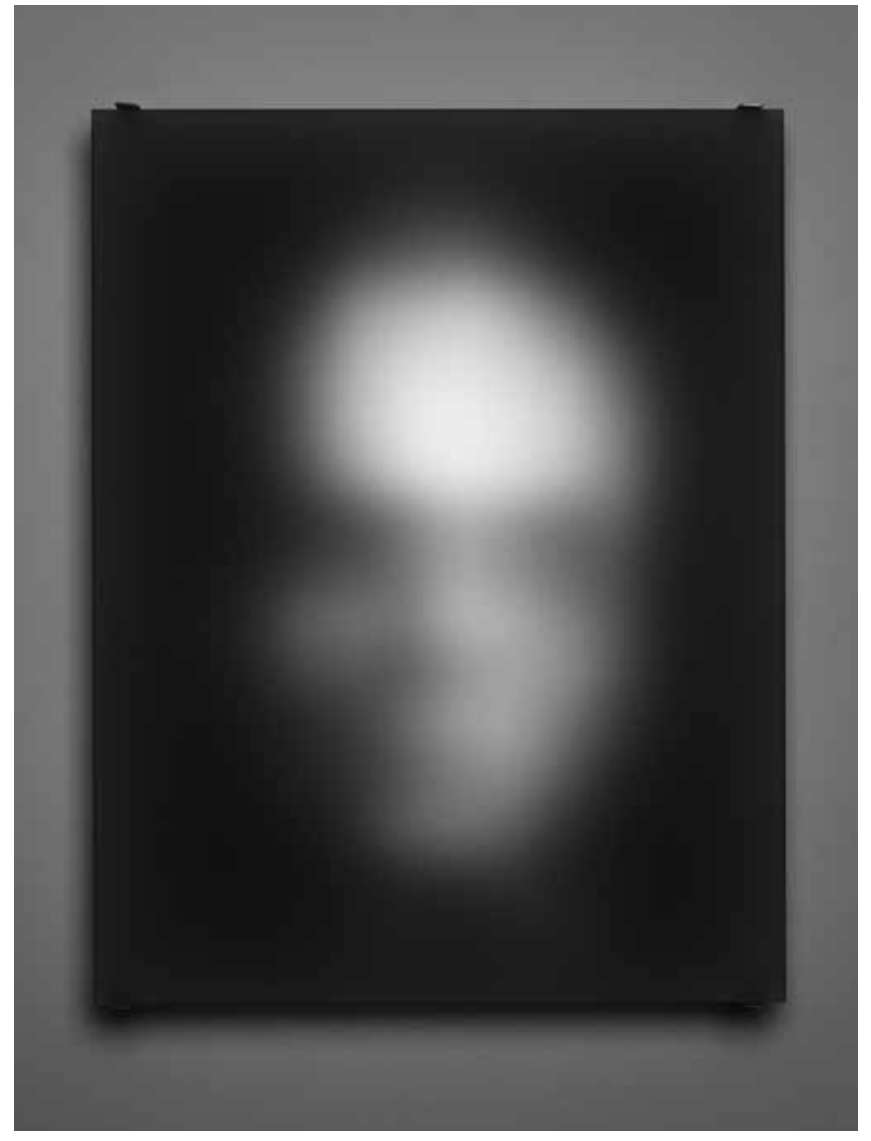

Figure I. Jim Campbell, Portrait of a Portrait of Harry Nyquist, 2000, matériel électronique, 192 diodes, plexiglas traité, $38,0 \times 30,5 \times 7,5 \mathrm{~cm}$, New York, Collection de Neuberger Berman LLC (photo : Sarah Christianson).

cognitif de l'information visuelle ${ }^{4}$, et la seconde, à travers une transposition exploratoire de la théorie de l'information à la sphère artistique, effectuée par Umberto Eco dans les années 19605. L'objectif de ces deux dernières parties consistera à appréhender les moyens mis en œuvre pour susciter une réaction chez le spectateur, sans présumer de leurs effets réels ni conjecturer sur l'expérience vécue par le public de Jim Campbell.

\section{Portrait d'une théorie}

Les portraits de Campbell se présentent comme deux dispositifs identiques, de très faible résolution, sur lesquels défile une séquence vidéo créée à partir d'anciennes photographies nécrologiques. Matériellement, les dispositifs sont composés d'un panneau métallique muni de 192 diodes lumineuses, devant lesquelles est placé un plexiglas semi-transparent (fig. 3 ) : le groupe de diodes forme une matrice de pixels qui retransmet en boucle la vidéo (une diode correspond à un pixel), tandis que le plexi-

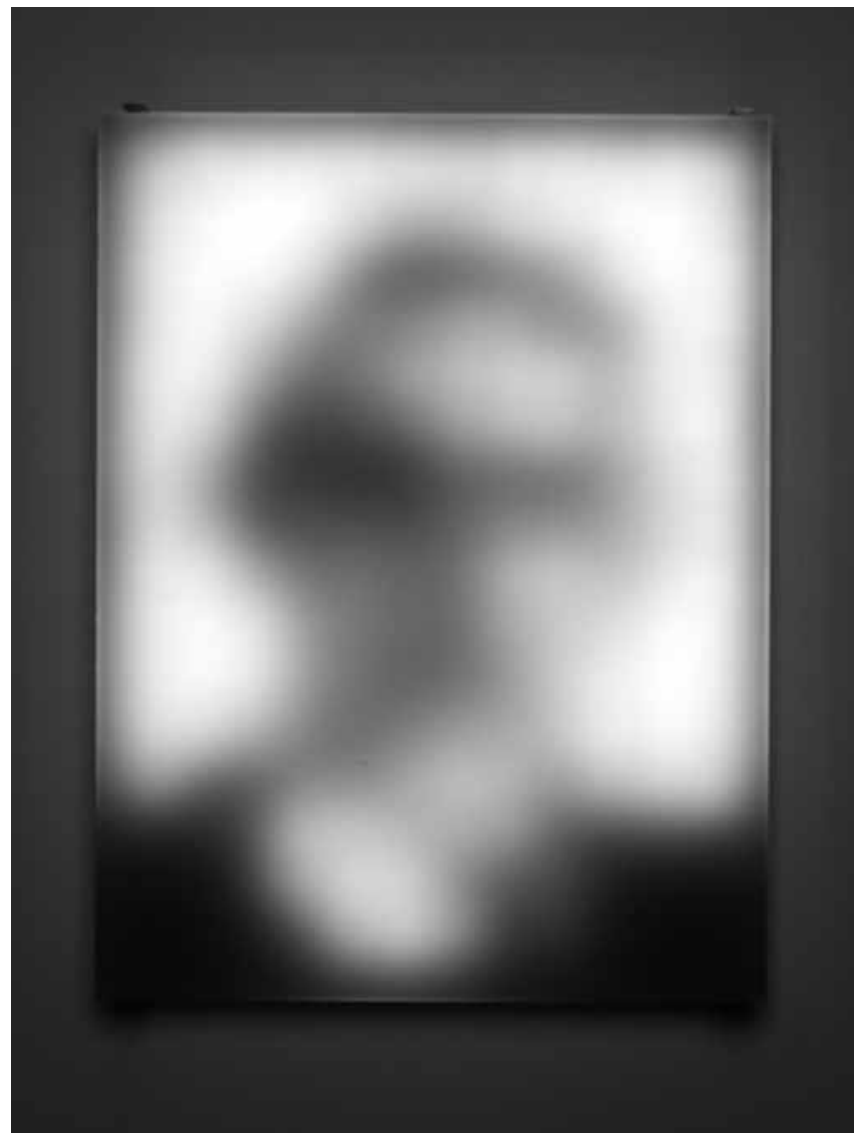

Figure 2. Jim Campbell, Portrait of a Portrait of Claude Shannon, 2000, matériel électronique, 192 diodes, plexiglas traité, $38,0 \times 30,5 \times 7,5 \mathrm{~cm}$, Austin, Austin Museum of Art (photo: Sarah Christianson).

glas voile partiellement cette matrice et filtre les variations de lumière, ce qui produit le résultat étonnant de faire apparaître l'image. Dans la séquence, les photographies sources sont comprimées jusqu'à la limite de la perceptibilité et des perturbations aléatoires, soit des bruits de nature visuelle qui entraînent une dégradation sporadique de l'image, viennent troubler la reconnaissance des visages à intervalles (fig. 4) ${ }^{6}$.

Les modèles représentés, Harry Nyquist (1889-1976) et Claude Shannon (1916-2001), sont deux scientifiques dont les travaux ont pavé la voie à une théorie qui constitue le fondement des technologies numériques actuelles, soit la théorie de l'information7. Celle-ci est un modèle de communication mathématique, créé en 1948 pour les télécommunications, en l'occurrence pour améliorer l'efficacité des réseaux téléphoniques, mais dont les applications s'étendent par la suite à diverses disciplines dont celle des sciences cognitives. Ce modèle scientifique se penche sur deux questions principales qui sont la compression et la transmission de données. En premier lieu, 


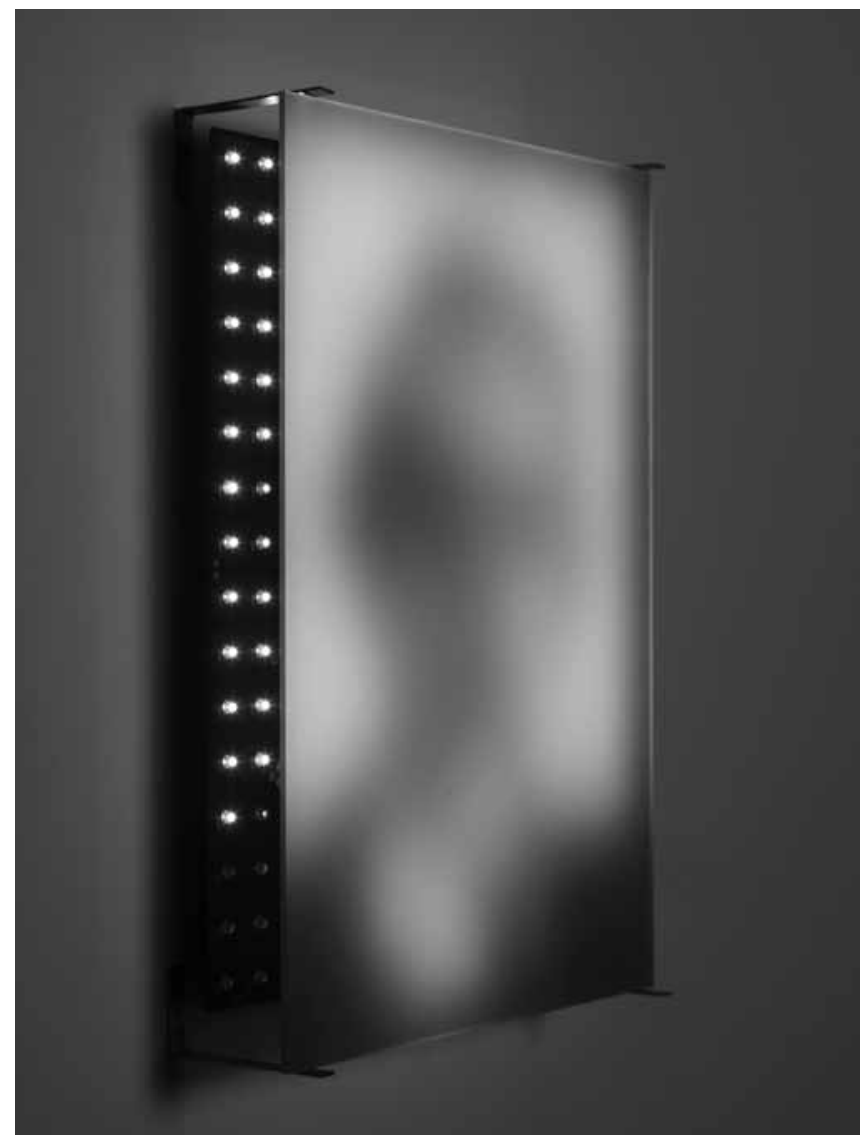

Figure 3. Jim Campbell, Portrait of a Portrait of Claude Shannon, 2000, matériel électronique, 192 diodes, plexiglas traité, $38,0 \times 30,5 \times 7,5 \mathrm{~cm}$, Austin, Austin Museum of Art (photo: Sarah Christianson).

il s'attache à la description succincte de l'information, c'est-àdire à sa compression, pour les besoins de mise en mémoire. En second lieu, il s'intéresse à la fiabilité de la transmission d'un émetteur à un récepteur à travers un canal bruyant, autrement dit à travers un support de transmission non fiable. En gros, Nyquist a établi qu'un signal vocal analogique peut être encodé mathématiquement et, à sa suite, Shannon a démontré qu'une information, peu importe qu'elle soit sonore, visuelle ou autre, peut être représentée sous forme binaire, c'est-à-dire quantifiée en bits ou réduite à une séquence de 1 et de 08 . Il s'ensuit que tout message peut être encodé, transmis par un émetteur, puis décodé par un récepteur sans perte significative de qualité, et ce, même si des perturbations parasitent le canal et que ledit message se corrompt en cours de route (fig. 5). De fait, l'encodage permet de régénérer le message à la réception et donc de le conserver à peu près intact. Lors de ce processus, l'information subit une double conversion : d'analogique qu'elle était au départ, elle devient numérique, pour redevenir analogique à la fin.

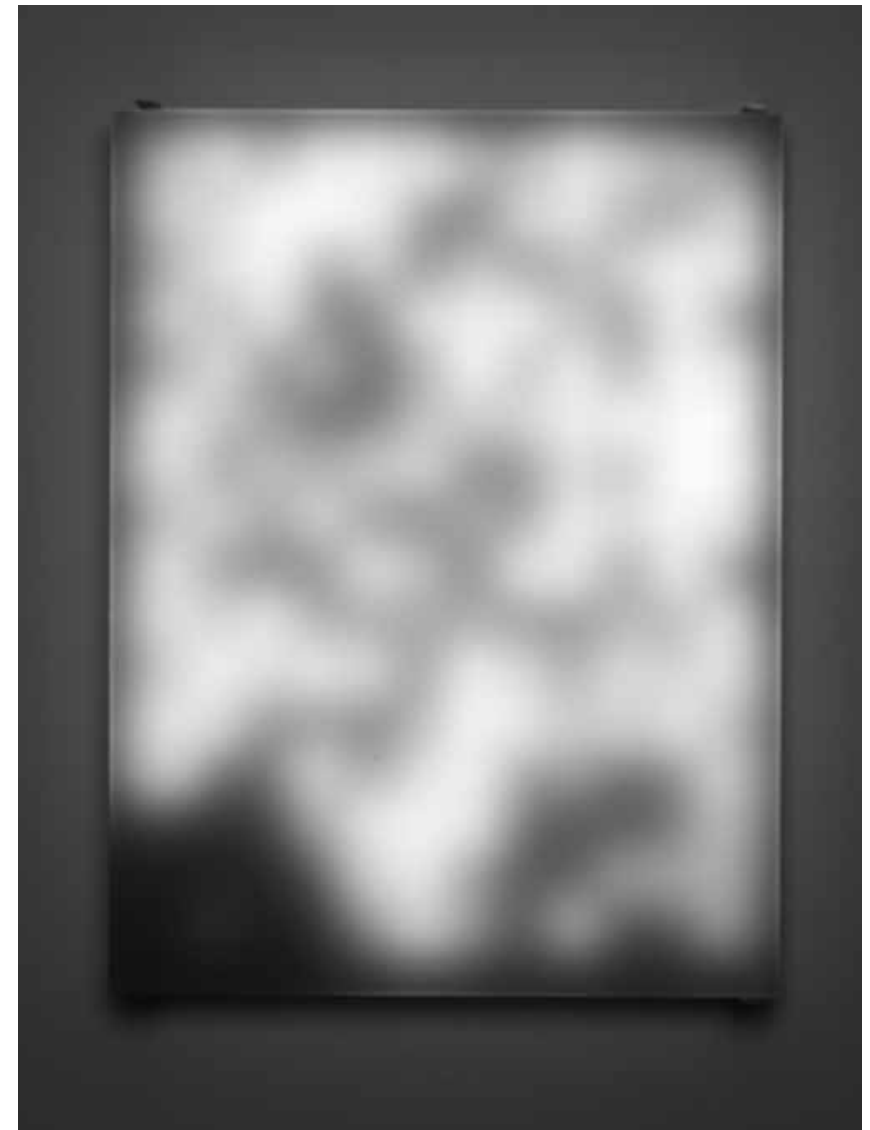

Figure 4. Jim Campbell, Portrait of a Portrait of Claude Shannon, 2000, matériel électronique, 192 diodes, plexiglas traité, $38,0 \times 30,5 \times 7,5 \mathrm{~cm}$, Austin, Austin Museum of Art (photo : Sarah Christianson).

Au moyen du médium électronique, Jim Campbell transpose visuellement et conceptuellement les deux applications de la théorie que sont la compression et la transmission. D'abord, il compresse l'image jusqu'à la rendre quasi imperceptible. L'information visuelle contenue dans la photographie nécrologique initiale est réduite à un groupuscule de 192 pixels. Ensuite, l'artiste fait référence au processus de transmission de deux manières, d'un côté, en effectuant une conversion analogiquenumérique-analogique, de l'autre, en brouillant l'image vidéo de façon cyclique. Ainsi, la photographie d'origine, qui est analogique, est transformée en vidéo numérique, puis l'écran de plexiglas est utilisé comme un filtre convertisseur qui retransforme la vidéo en image analogue à la photographie source. La formulation des titres, "portrait of a portrait ", soulignerait alors ces remédiations successives, ces mutations d'un médium à un autre qui posent les œuvres moins en tant qu'images d'un modèle qu'en tant qu'images d'images 9 . Dans la même veine, le brouillage intermittent du visage, précédé et suivi de sa 
réapparition, suggérerait la transmission en désignant tour à tour l'intégrité du message à l'émission, sa corruption lors du passage à travers un canal bruyant et sa reconstitution à la réception. En somme, les portraits de Nyquist et Shannon reproduisent, par leur aspect visuel et leur conception matérielle, le modèle de la théorie de l'information et ses deux principales applications technologiques.

\section{Portrait de l'esprit}

Par leur référence à la théorie de l'information, les portraits de Campbell évoqueraient non seulement un processus de traitement de données, au sens informatique du terme, mais aussi un processus de cognition humaine. Ils représenteraient autant un modèle de communication mathématique appliqué aux sciences informatiques qu'un modèle traditionnel des sciences cognitives. Cette interprétation est étayée par les productions antérieures de l'artiste, notamment par la série Memory Works (1994-1998) qui, dans le même ordre d'idées, établit un rapprochement entre mémoire informatique et mémoire humaine. Elle est aussi en partie confirmée par l'artiste dans la proposition de recherche qu'il soumet à la Fondation Daniel Langlois : "Using technological tools and scientific models as metaphors for memory and illusion, my work seeks to interpret, represent and mirror psychological states and processes, and their breakdown ${ }^{10}$. De fait, le système cognitif humain est perçu assez souvent comme un système de traitement actif de l'information sensorielle. La théorie de l'information a apporté une contribution majeure à cette conception en lui fournissant un cadre théorique : le modèle de traitement de l'information a été transposé du domaine des communications et de l'informatique à celui de la cognition ${ }^{11}$. Le philosophe Mark Rowlands décrit brièvement la conception du système cognitif qui découle de cette transposition et la situe dans le champ des sciences cognitives :

The idea that cognition involves information processing is now commonplace. Indeed, it is a central plank of the conception of cognition embodied in the cognitive science tradition. In its classical form, cognitive science was understood to involve the postulation of internal configurations of an organism or system : configurations that carry information about extrinsic states of affairs. The concept of information employed is, in essence, that elaborated by Claude Shannon (1948), or a close variant thereof 12 .

Le modèle de cognition, issu des théories de Shannon, se fonde sur une mise en parallèle de l'humain et de l'ordinateur et constitue, comme le mentionne Rowlands, un modèle classique des sciences cognitives ${ }^{13}$. Il procède d'une approche traditionnelle qui n'est ni généralisée à l'ensemble de la discipline ni exempte de critiques. Cette conception serait en effet lacunaire,

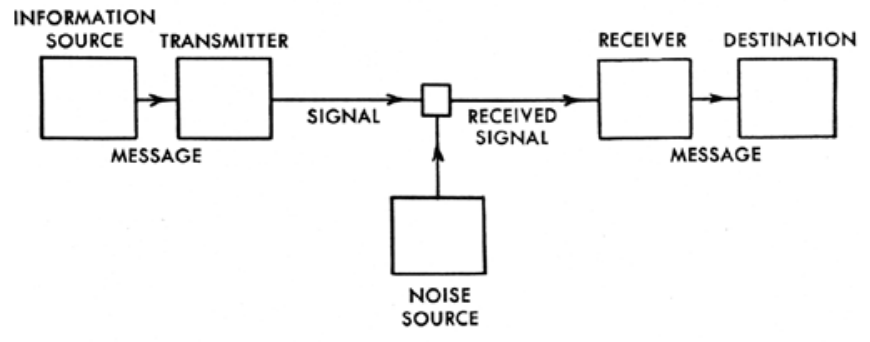

Figure 5. Système général de communication, 1948 (photo : dans Claude E. Shannon, «A Mathematical Theory of Communication », The Bell System Technical Journal, vol. XXVII, juillet/octobre 1948).

car elle négligerait, tel que l'exprime le philosophe Pierre Steiner, les "propriétés conscientes, somatiques, temporelles, environnementales, dynamiques et sociales de la cognition "14. À la vérité, ce modèle scientifique, dit internaliste, tend à nier toute interdépendance entre le sujet percevant et son environnement. Sa validité est sans doute discutable à plusieurs égards, mais elle importe peu pour les besoins de la présente argumentation, puisque l'objectif est ici de montrer que les portraits reproduisent bel et bien un modèle de cognition.

La prémisse du modèle basé sur la théorie de l'information est que le système cognitif interprète les stimuli à la manière dont un système informatique traite les données : il les recevrait, les manipulerait, les emmagasinerait et les récupérerait de la même façon. Cette analogie a donné naissance à une approche nommée " approche de traitement de l'information " (information-processing approach) ${ }^{15}$. Celle-ci conçoit l'exécution des processus mentaux, notamment de la perception sensorielle, comme une séquence d'opérations indépendantes, successives ou parallèles, s'apparentant à celles réalisées par un programme informatique ${ }^{16}$. Plus spécifiquement, les étapes du traitement de l'information perceptuelle par l'humain ressembleraient aux opérations logiques qu'effectue un algorithme intégré dans un programme pour résoudre un problème donné17. D'après ce modèle des sciences cognitives, l'algorithme et, par extension, le programme sont tenus pour des analogues de l'esprit humain, car ils posséderaient comme lui une fonction de traitement de l'information. Dès lors, selon cette conception, l'esprit fonctionnerait sur le mode de ce qui peut être appelé une "pensée algorithmique ».

Les travaux scientifiques d'Harry Nyquist et de Claude Shannon ont donné lieu à la formule d'interpolation NyquistShannon, un algorithme sur lequel repose le théorème d'échantillonnage Nyquist-Shannon. Celui-ci se penche sur la conversion entre signaux analogiques et numériques et a été défini par Shannon, en 1949, dans un article intitulé "Communication in the Presence of Noise ${ }^{18}$. Dans ses grandes lignes, le texte 


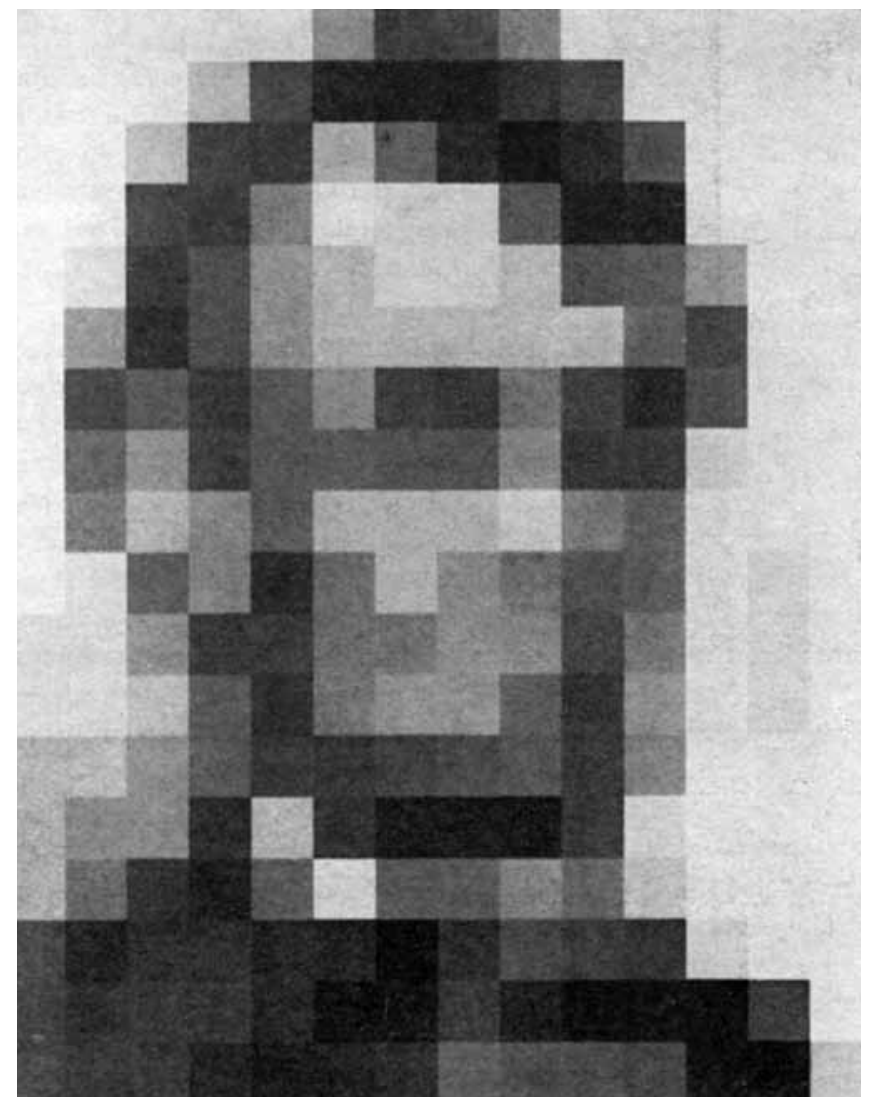

Figure 6. Leon D. Harmon, Abraham Lincoln, 1973, impression numérique, [dimensions et collection non disponibles] (photo : dans Leon D. Harmon, «The Recognition of Faces », Scientific American, vol. CCXXIX, n 5, 1973. Reproduit avec la permission d'Alcatel-Lucent USA Inc.).

reformule d'un point de vue technique les éléments fondamentaux de la théorie de l'information, exposés en termes mathématiques l'année précédente dans «A Mathematical Theory of Communication ${ }^{19}$. L'algorithme décrit les transformations infimes apparues lors de la conversion analogique-numérique, essentiellement la perte d'information, et ce, dans l'intention d'optimiser l'efficacité de la transmission en présence de bruit. Le fonctionnement cognitif serait donc évoqué dans les œuvres d'art par la référence générale à la théorie de l'information, mais également par la référence spécifique à l'algorithme portant le nom des deux scientifiques.

\section{Portrait d'une expérience perceptuelle}

Si un modèle théorique de cognition est représenté de manière visuelle et conceptuelle à l'intérieur des œuvres, le processus cognitif, quant à lui, est vécu à l'extérieur de celles-ci chez le spectateur. Dans un jeu de mise en abîme, le traitement cognitif de l'information s'inscrit donc à l'intérieur des portraits en tant que représentation et à l'extérieur de ceux-ci en tant qu'expérience. Dans ce dernier cas, pour reprendre le modèle internaliste, les portraits émettent une information visuelle qui est traitée, stockée et récupérée par un récepteur : le spectateur. Ainsi, Jim Campbell réinterprète une expérience scientifique exécutée en 1973 par Leon Harmon, un ingénieur et un artiste qui, comme Nyquist et Shannon, a travaillé, à un moment ou à un autre, pour les Bell Telephone Laboratories ${ }^{20}$. Plus précisément, l'artiste reproduit, dans l'ensemble de la série "Ambiguous Icons ", un phénomène perceptuel qui a été étudié et documenté par Harmon. Au moins deux sources attestent de l'appropriation. D'une part, dans le catalogue d'une exposition tenue à Santa Fe, en 2005, le commissaire Steve Dietz y fait référence en examinant un autoportrait de la série, à savoir I Have Never Read the Bible Again (2001). D’autre part, Jim Campbell décrit quelques essais préliminaires en ce sens dans le projet qu'il rédige pour la Fondation Daniel Langlois ${ }^{21}$. À n'en pas douter, la similitude entre l'expérience scientifique et les portraits est d'autant plus saisissante que l'ingénieur axe son étude sur la représentation du visage humain.

En s'appuyant sur la théorie de l'information, Harmon réalise effectivement plusieurs portraits hautement pixélisés, notamment une manipulation d'un portrait d'Abraham Lincoln, afin d'étudier la reconnaissance faciale dans des conditions où l'information visuelle est très limitée ${ }^{22}$. Il publiera les résultats complets de sa recherche la même année dans un article intitulé "The Recognition of Faces »23. L'ingénieur cherche à savoir dans quelle mesure la reconnaissance est altérée lorsque l'image d'un visage est floue ou dégradée 24 . Pour y parvenir, il réduit quantitativement l'information contenue dans des portraits photographiques : il numérise les images, puis en abaisse considérablement la résolution, ce qui produit, pour reprendre son expression, des block portraits, c'est-à-dire des portraits constitués d'un assemblage semi-abstrait d'immenses carrés, ou pixels, de diverses valeurs de gris (fig. 6). Le but recherché est de quantifier l'information minimale requise à la reconnaissance faciale. Harmon expose ainsi sa démarche expérimentale :

[A] way to study recognition is to ask how little information, in the informal sense of " bit, " or binary digits, is required to pictorially represent a face so that it can be recognized out of a finite ensemble of faces. We explored this «threshold " of recognition with portraits that had been precisely blurred 25 .

L'ingénieur cherche, somme toute, à chiffrer un "seuil " situé à la limite de la reconnaissance et de la non-reconnaissance. La démarche présuppose l'avènement d'un changement brusque, statistiquement généralisable, entre capacité et incapacité à reconnaître. Les scientifiques remettent aujourd'hui en doute l'existence d'un tel seuil dans l'expérience sensorielle et 
considèrent plutôt que la réaction de l'observateur s'effectue graduellement selon des facteurs tant objectifs que subjectifs, en l'occurrence de nature physiologique et psychologique ${ }^{26}$. Quoi qu'il en soit, la démarche n'est pas étrangère à celle de Campbell, puisque ce dernier s'efforce lui aussi de déterminer la plus basse résolution à laquelle l'œil peut percevoir un visage humain : "I wanted to experiment with the idea of taking the smallest amount of information (pixels) where you (the human mind) could still perceive something $" 27$. Ainsi, en ce qui touche la quantification de l'expérience perceptuelle, les objectifs de Harmon et Campbell apparaissent tout à fait identiques et, au surplus, leurs résultats numériques se révèlent similaires. L'enquête de Harmon démontrera qu'une résolution approximative de 256 pixels, soit 16 x 16 " carrés ", représente le seuil minimal permettant l'identification des visages ; Campbell chiffrera ce même seuil à 192 pixels, soit le nombre de diodes intégrées à ses dispositifs vidéographiques ${ }^{2}$.

Les block portraits posent un défi aux scientifiques qui étudient les phénomènes de perception visuelle, car, contre toute attente, leur observation à distance facilite la reconnaissance des physionomies. En d'autres termes, les visages sont plus aisément identifiables de loin, ce qui s'avère contre-intuitif. De fait, comme l'explique Harmon, la distance entre l'image comprimée et l'observateur crée l'équivalent d'un flou qui accroît le degré de perceptibilité : " recognition can be enhanced by viewing the picture from a distance, by squinting at it, by jiggling it or by moving the head while looking at it. The effect of all these actions is to blur the already degraded image $" 29$. L'ingénieur avance deux hypothèses qui pourraient expliquer scientifiquement ce phénomène. Ces hypothèses partent du principe que toute image peut être décrite comme un ensemble de fréquences optiques et que le flou, créé par l'éloignement, réduit certaines fréquences parasites, des bruits, qui tendent à masquer les visages et donc à limiter la reconnaissance faciale.

La première hypothèse implique que les carrés parsemant le portrait de Lincoln, avec leurs arêtes vives, soient l'équivalent optique d'une haute fréquence sonore et que la haute fréquence, tant optique que sonore, nuise à la perceptibilité. Les formes géométriques seraient alors une interférence de haute fréquence qui dissimule les visages. Le postulat suppose qu'en plaçant devant les block portraits un filtre qui, en langage simple, rend l'image floue ou, en langage scientifique, élimine les hautes fréquences, la visibilité serait améliorée (fig. 7). Les effets de la défocalisation obtenue par filtrage seraient à cet égard identiques à ceux de l'observation à distance. Jim Campbell effectue une procédure semblable : le panneau de diodes, en tant que matrice pixélisée, fait office de block portrait devant lequel l'artiste place un plexiglas traité qui agit tel un filtre (fig. 8). Selon le raisonnement de Harmon, ce plexiglas, en diffusant la lumière, briserait visuellement la structure des diodes-

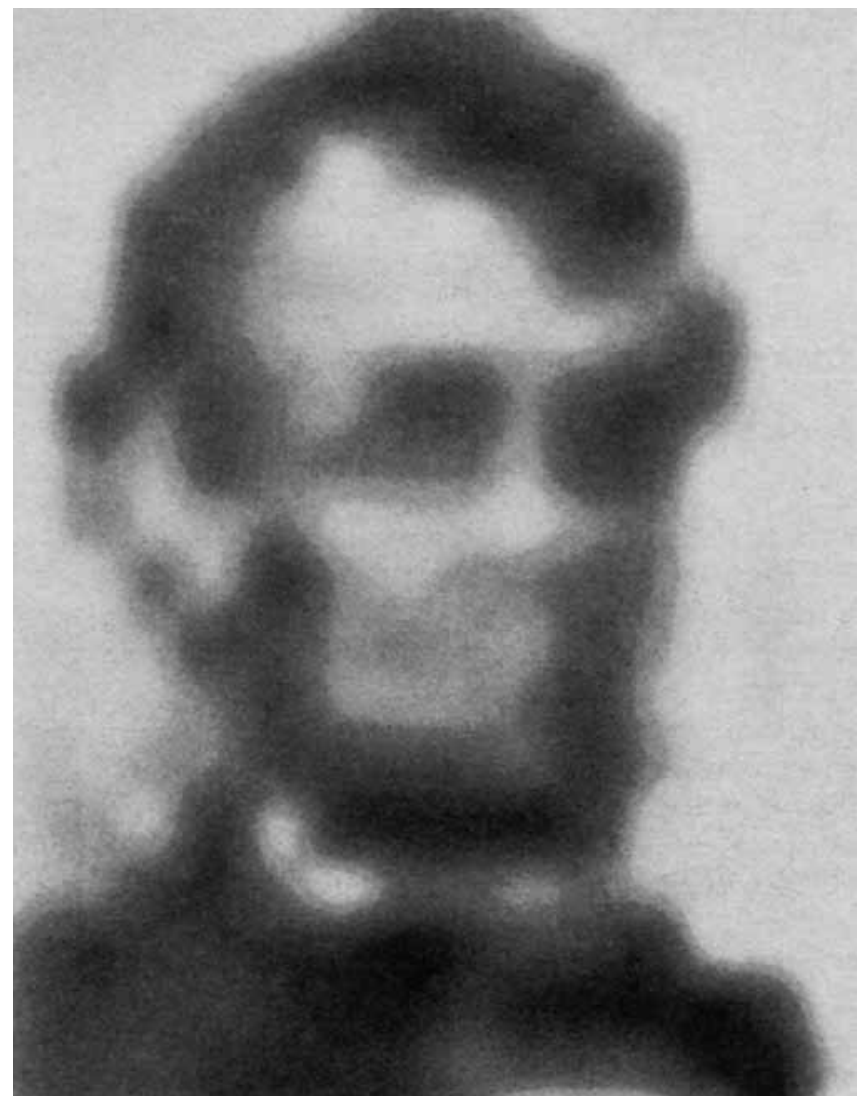

Figure 7. Leon D. Harmon, Abraham Lincoln [après filtrage], 1973, impression numérique, [dimensions et collection non disponibles] (photo : dans Leon D. Harmon, «The Recognition of Faces », Scientific American, vol. CCXXIX, $n^{\circ}$ 5, 1973. Reproduit avec la permission d'Alcatel-Lucent USA Inc.).

pixels, soit les hautes fréquences optiques, ce qui augmenterait la perceptibilité de l'image. C'est ainsi que, grâce au filtrage, les visages de Nyquist et de Shannon pourraient apparaître, alors que, sans lui, seules des variations lumineuses abstraites demeureraient visibles ${ }^{30}$.

En seconde hypothèse, Harmon propose que le bruit optique nuisant à la lisibilité appartienne surtout à un spectre de fréquences proche de celui de la photographie originale de Lincoln. L'explication est que, comme dans le cas du son, la proximité de la fréquence d'un bruit indésirable avec un signal porteur d'information tend à compromettre la détection de ce dernier. Il y aurait donc une bande critique de fréquences qui voilerait encore davantage les physionomies que ne le font les hautes fréquences. Afin de vérifier cet autre postulat qu'il nomme critical-band masking, Harmon réalise une double opération : il réduit d'abord la définition de la photographie par un flou uniforme, plus précisément, il la filtre selon certaines spécifications scientifiques; puis, il superpose sur cette image voilée des 
bruits aléatoires de différentes fréquences. Lorsqu'un bruit de fréquences adjacentes à celles du portrait source est introduit, le visage se brouille de façon évidente, alors qu'il demeure relativement identifiable lorsque les fréquences sont plus éloignées (fig. 9). D'après les conclusions de l'étude, ce résultat prouverait que, dans des conditions où l'image est dégradée, le criticalband masking est la principale cause de non-reconnaissance. Grossièrement résumée, dans cette dernière expérimentation, la démarche consiste à ajouter des bruits aléatoires de différentes fréquences sur un portrait rendu flou par filtrage. Dans ses portraits électroniques, Campbell met en place une procédure qui combine alors les deux expériences de Harmon : il construit l'équivalent de block portraits avec un ensemble de diodes; il les filtre avec un plexiglas, ce qui fait émerger des visages diffus ; puis, il introduit des bruits aléatoires qui brouillent périodiquement la représentation. Seulement, au lieu de concevoir une série d'images statiques, l'artiste produit des images en mouvement et juxtapose, en une seule séquence vidéo, les différentes opérations exécutées par le scientifique. En un mot, il ne reproduit pas à l'identique les expérimentations, mais les adapte en exploitant les potentialités de son médium, dans un but qui, par ailleurs, dépasse les seules considérations scientifiques.

Les points de comparaison établis avec les expérimentations de Harmon révèlent les motivations scientifiques sous-jacentes au processus de création de Campbell et exposent, du moins en partie, la genèse des œuvres d'art. Mais vue uniquement sous cette loupe, l'expérience des portraits se résumerait à une activité de reconnaissance faciale mesurée en résultats quantifiables. Dans les faits, la compréhension des phénomènes optiques qui régissent les portraits électroniques ne rend compte que d'une seule facette de la réponse cognitive, quoique la plus immédiate dans le cas du portrait : l'identification des visages. Comme le suggère le commissaire Steve Dietz à propos de l'autoportrait I Have Never Read the Bible Again, si les travaux de Campbell explorent bien les limites de la perception, leur raison d'être déborde l'horizon de cette investigation :

If Campbell's work were only about the limits of perception, what differentiates it from the information theory and scientific visualization work at Bell labs that sparked the investigation ? How is I Have Never Read the Bible Again (2001) better or worse than or ever different from Leon Harmon's 1973 conversion of a picture of Abraham Lincoln into large, discrete pixels, which, when viewed at a distance, coalesce into a fuzzy but more continuous and recognizable portrait ${ }^{31}$ ?

À l'évidence, la proposition artistique ne s'inscrit pas en substitut de l'expérimentation scientifique. Elle s'avère plutôt une appropriation, issue du domaine de la science, qui explore les effets de l'ambiguïté perceptuelle, poussée à son extrême limi- te, sur l'expérience esthétique. Or la théorie de l'information pourrait nous éclairer à ce sujet, non sans quelques précautions, puisqu'elle étudie la communication dans sa définition élargie. Il s'agira donc, dans le développement de notre propos, d'esquisser à grands traits une transposition théorique entre la science et les arts, une transposition qui certes n'est pas nouvelle, et d'apprécier dans quelle mesure celle-ci peut favoriser la compréhension des stratégies mises en place pour orienter l'expérience esthétique.

\section{Portrait d'une expérience esthétique}

Au tournant des années 1960, la théorie de l'information suscite un engouement, tantôt durable, tantôt passager, dans les domaines qui touchent de près ou de loin celui de la communication. Dans le sillage de l'enthousiasme qu'elle provoque alors, notamment en psychologie expérimentale, des transpositions théoriques sont tentées, de façon exploratoire, dans l'étude de la perception esthétique ${ }^{32}$. Ces recherches, qui abordent autant l'observation visuelle que l'écoute musicale ou la lecture poétique, sont loin de faire l'unanimité, surtout en raison des apories liées au fondement mathématique de la théorie. En guise d'exemple, le psychologue de la perception et théoricien de l'art Rudolf Arnheim (1904-2007), qui préside pourtant en 1957 un colloque consacré aux rapports que noue la théorie de l'information avec les arts 33 , affirmera près d'une quinzaine d'années après l'événement : " the tempting prospect of applying information theory to the arts and thereby reducing aesthetic form to quantitative measurement has remained largely unrewarding $" 34$. Pour tout dire, si la théorie de l'information laisse bel et bien une empreinte profonde dans le champ des sciences cognitives, elle a donné lieu, dans le champ de l'art, à des réflexions d'un intérêt variable dont les retombées se seront révélées, pour la plupart, de courte durée.

Néanmoins, parmi les quelques recherches n'ayant pas succombé à un réductionnisme mathématique, celle que publie en 1962 Umberto Eco dans le troisième chapitre d'Opera aperta (L'œuvre ouverte) a sans contredit trouvé des oreilles attentives, au point où la transposition à la sphère artistique, une transposition quelque peu fidèle s'entend, n'aura vraisemblablement perduré de nos jours qu'à travers le travail de cet auteur 35 . Sans truffer son analyse d'équations impénétrables, mais sans non plus occulter la définition mathématique de la théorie, Eco se penche sur la réponse induite par les œuvres modernes de son temps en appliquant, avec des ajustements indispensables, les principes généraux du modèle de communication conçu par Claude Shannon. Il théorise ainsi l'expérience esthétique en tant qu'acte communicationnel, sans se fier à une mesure quantifiée ni dénaturer le modèle scientifique. Son analyse se penche, à l'instar de la théorie de l'information, sur l'efficacité 

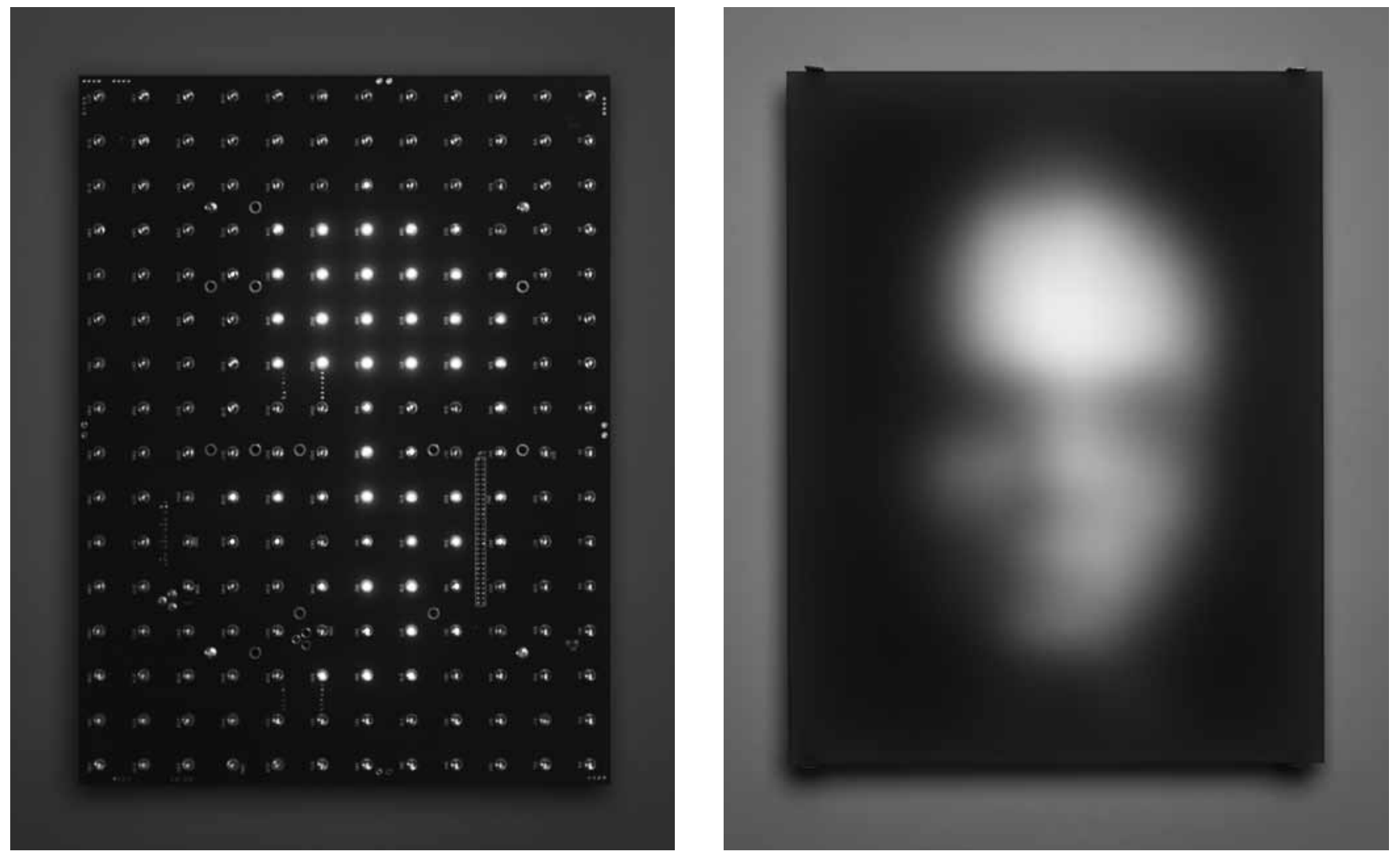

Figure 8. Jim Campbell, Portrait of a Portrait of Harry Nyquist, 2000, matériel électronique, 192 diodes, [sans et avec] plexiglas traité, 38,0 × 30,5 × 7,5 cm, New York, Collection de Neuberger Berman LLC (photo : Sarah Christianson).

de la "transmission " et non sur la valeur ou la signification du " message " transmis. Elle s'intéresse donc à la communication artiste-œuvre-spectateur (émetteur-message-récepteur), particulièrement à la dynamique œuvre-spectateur, et non à l'œuvre d'art en tant que donnée autonome, que ce soit sur le plan formel ou sur le plan conceptuel. L'auteur articule sa réflexion autour d'une notion qu'il élabore à travers l'ouvrage entier et qu'il nomme l'" ouverture ". Le recours à la théorie de l'information s'apparente alors à une exploration méthodologique, à la recherche d'un appareil théorique, permettant d'aborder la question de l'ouverture dans les œuvres contemporaines, c'est-à-dire, pour Eco, les œuvres du début des années 1960. Pour ainsi dire, la démarche reste ouverte et, en vérité, l'intérêt qu'Eco porte à la théorie de l'information le mènera éventuellement vers d'autres approches théoriques. Quoi qu'il en soit, Jim Campbell s'intéresse lui aussi, avant toute chose, au processus de communication et ses portraits, même s'ils appartiennent à un contexte culturel bien différent, ne sont pas sans partager les propriétés communicationnelles associées au concept d'ouverture. Pour s'en convaincre, il suffira de résumer brièvement l'ar- gumentation d'Eco sur le sujet, puis de se tourner vers la théorie de l'information et vers le cadre qu'elle offre pour analyser l' " œuvre ouverte».

Eco commence la préface de L'œuvre ouverte en affirmant que "l'œuvre d'art est un message fondamentalement ambigu " 36 . L’ambiguïté, selon lui, serait une condition inévitable de l'œuvre d'art, car elle participerait de toute communication humaine. Cependant, dans le contexte contemporain, cette ambiguïté implicite se transformerait en un but explicite à atteindre : les artistes s'attacheraient à la cultiver pour en faire un "principe de création ". Ils recourraient "à l'informel, au désordre, au hasard, à l'indétermination " dans l'intention de produire, délibérément, une ouvre dont la marque est de faire appel à la contribution active du spectateur, de déployer un espace de suggestion qui sollicite son imagination, ses émotions, sa sensibilité, et qui, par la même occasion, multiplie les pistes interprétatives.

Afin d'expliciter le concept d'ouverture et l'étendue de ses effets, Eco analyse les réactions d'un auditeur à différents stimuli, en l'occurrence à trois propositions linguistiques, correspondant respectivement à ce qu'il appelle la "référence », la « suggestion » 

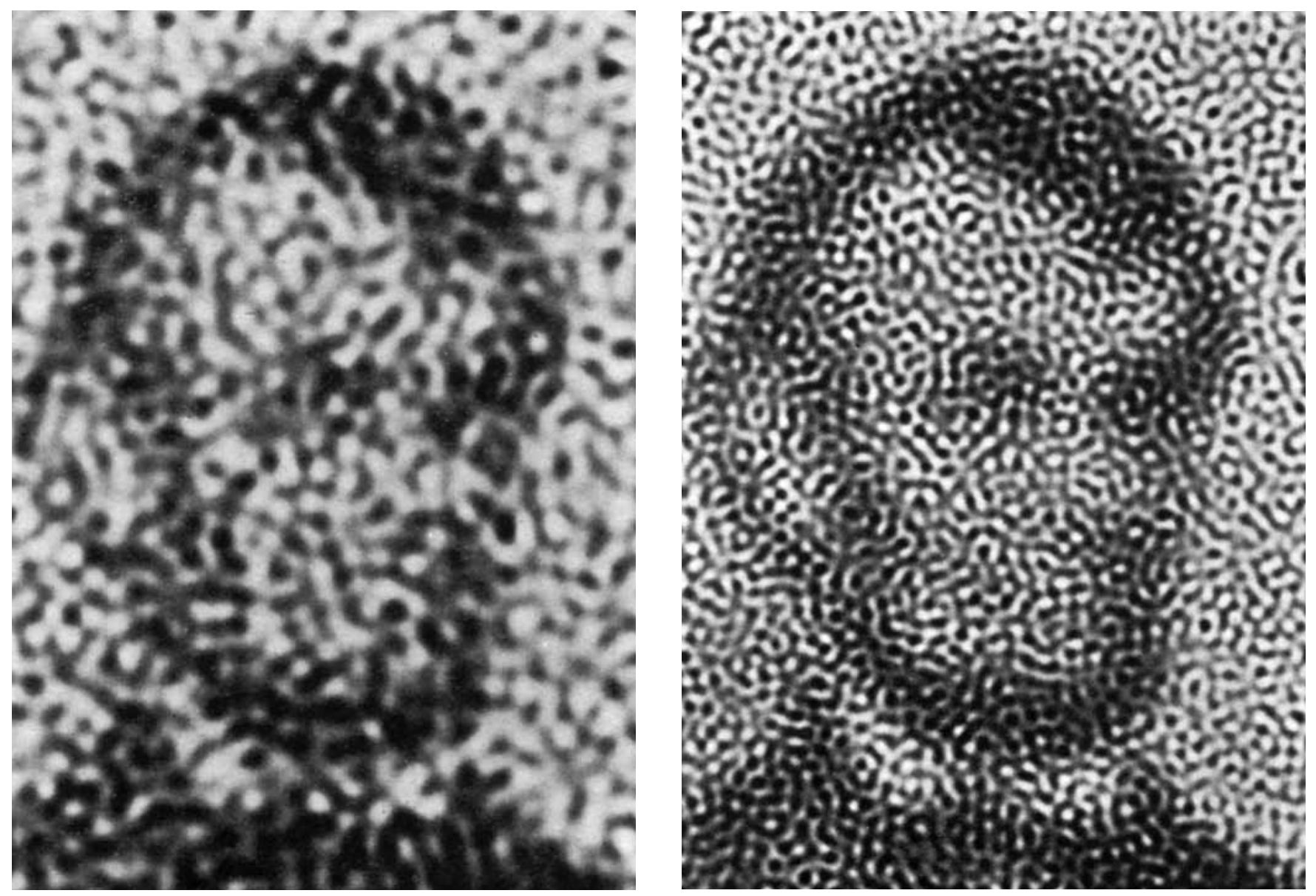

Figure 9. Leon D. Harmon, Abraham Lincoln [avec bruits de fréquences adjacentes et de fréquences éloignées], 1973, impression numérique, [dimensions et collection non disponibles] (photo: dans Leon D. Harmon, « The Recognition of Faces », Scientific American, vol. CCXXIX, n 5, 1973. Reproduit avec la permission d'Alcatel-Lucent USA Inc.).

et la «suggestion dirigée ». La démonstration, quoique restreinte au langage, vaut pour tout type de stimulus et, dans notre interprétation, elle tend à confirmer que Campbell joue explicitement de l'ouverture propre à la suggestion dirigée. Ainsi, dans un premier temps, Eco note qu'une expression référentielle, aussi précise et conventionnelle soit-elle, ce qu'il désigne comme étant la "référence ", porte nécessairement une part d'ouverture dans la mesure où elle est communiquée à un destinataire. Il va sans dire que ce dernier peut, selon sa culture, ses connaissances, voire son humeur, teinter la proposition d'une infinité de nuances particulières. Pourtant, cette ouverture incontournable ne rompt pas l'univocité première du message : le contenu référentiel, même variablement connoté, demeure identique pour tout destinataire. Il en irait toutefois autrement pour l'ouverture donnée par la "suggestion ». Dans un deuxième temps, Eco soutient effectivement qu'une expression référentielle imprécise, en raison même de son imprécision, peut, selon l'auditeur à qui elle s'adresse, être comprise de plusieurs façons. Il en conclut que "la différence référentielle [...] ne réside pas dans l'expression mais dans le récepteur $" 37$. Reste cependant que la suggestion demeure désorganisée, sans objet, et que, comme le remarque Eco, son effet sur l'auditeur tendra à s'épuiser rapidement. Pour produire un impact plus durable et plus sûr, elle devrait plutôt être orientée grâce à une organisation des stimuli, c'est-à-dire une organisation matérielle et formelle. L'auteur examine donc, dans un troisième temps, la réponse à une expression référentielle imprécise enrichie d'associations sonores : la "suggestion dirigée ». Dans son hypothèse, l'ajout délibéré de suggestions phoniques à une indétermination référentielle créerait un effet " esthétique ", au sens large du terme, qui décuplerait l'efficacité de la proposition linguistique. Autrement dit, l'articulation entre le stimulus sensible et la référence indéfinie permettrait d'amplifier et, 
surtout, de diriger la réponse de l'auditeur à l'intérieur de paramètres circonscrits ou, pour reprendre l'expression, à l'intérieur d'un " champ de suggestions ».

Les suggestions sont voulues, provoquées, appelées dans les limites déterminées par l'auteur ou plus exactement par la machine esthétique qu'il a mise en mouvement. Cette machine n'ignore pas les capacités personnelles de réaction des spectateurs ; au contraire, elle les fait intervenir, elle y voit même la condition de son fonctionnement et de sa réussite : mais elle les oriente et les domine ${ }^{38}$.

Comment Jim Campbell, dans les portraits de Nyquist et Shannon, se servirait-il de la suggestion dirigée ? Par quels procédés matériels et visuels tenterait-il d'induire les effets de celle-ci chez le spectateur ? Si l'ouverture, pour lui, procède d'un principe de création artistique, ce qui est ici l'hypothèse, elle découle alors forcément d'une intention. À partir de là, il apparaît opportun d'examiner les dires de l'artiste quant à la réponse spectatorielle qu'il souhaite provoquer et aux moyens qu'il utilise pour parvenir à ses fins, et ce, tout en concédant qu'un monde sépare parfois les motivations initiales de leur impact final, car, cela va de soi, l'intention n'est jamais garante du résultat.

Very low resolution images exist at the borderline of abstraction. By reducing or eliminating the digital structure of an image, viewers are forced to search elsewhere in the image for meaning, causing color, motion and form to take on a new, more interdependent relationship with the interpretation of the image. In these works, as in other forms of visual abstraction, associative thinking processes play a larger role that linear or narrative thinking in the interpretation of the image. Understanding and using the characteristics of this process within an artistic methodology allows for unique forms of artistic expression while using only minute amounts of information. This is particularly true when the visibility of the raw information structure is eliminated [nous soulignons] ${ }^{39}$.

Ce sont les conclusions du rapport final déposé à la Fondation Daniel Langlois en août 2001. Elles confirment que, dans les images réalisées lors du projet, la basse résolution ainsi que la dissolution de la structure pixélisée sont explicitement employées dans le but de stimuler un mode de pensée par associations. En situant les œuvres aux limites de la figuration et de l'abstraction, ce qui, pour les portraits, correspond au seuil entre reconnaissance et non-reconnaissance, l'artiste produit une imprécision à la fois référentielle et formelle qui n'est pas fortuite comme dans la suggestion simple, mais précisément contrôlée comme dans la suggestion dirigée, contrôlée d'ailleurs au point où elle est quantifiée au nombre de pixels près. À ce propos, Umberto Eco parle de « la précision du mécanisme qui invite à l'imprécision " 40 .

La théorie de l'information, dans son étude de la communication, offre un cadre qui peut servir à explorer l'ouverture particulière causée par la suggestion dirigée. Sans présumer de l'incontestabilité de l'approche, il semble toutefois que ce cadre théorique, faisant écho à la référence scientifique introduite par l'artiste, permet d'appréhender les méthodes retenues pour infléchir la réponse spectatorielle. Dans l'adaptation qu'il en propose, Eco teinte son analyse, à un certain degré, de la vision moderniste propre au milieu intellectuel dans lequel il baigne, mais ce, sans appuyer un modernisme qui chercherait à isoler l'œuvre d'art de son spectateur ou de son contexte. Pour lui, les ouvres modernes existent par et pour un destinataire actif et elles tendent à évoquer, métaphoriquement, une conception du monde qui leur est contemporaine, notamment une conception scientifique. En fait, les traits du modernisme auxquels Eco souscrit concernent surtout la dimension sensible de l'œuvre, la configuration des stimuli, en d'autres termes, la " forme ". Sans adhérer au formalisme, Eco s'attache néanmoins à l'idée de rupture formelle (rupture d'un ordre, d'une structure, d'une norme), ce qui renvoie à la notion d'originalité et, implicitement, à une idéologie progressiste, une idéologie pourtant rejetée par l'auteur lui-même. Toute pensée n'est pas sans véhiculer sa part de contradictions et, en cela, celle d'Eco diffère à peine d'un certain postmodernisme qui substitue, aujourd'hui encore, l'idée de radicalité à celle de rupture et qui y décèle une transgression salutaire, un dépoussiérage bénéfique, bref un progrès. En substance, la transposition constitue un outil théorique imparfait, mais tout de même utile pour l'étude des portraits de Jim Campbell. Elle fournit des pistes de réponse quant à la démarche de ce dernier, d'une part, parce que les œuvres réfèrent directement à la théorie de l'information et, d'autre part, parce qu'elles partagent l'essentiel des caractéristiques de l'œuvre dite ouverte. Qui plus est, même si la transposition date d'il y a cinquante ans, la théorie de l'information possède une actualité renouvelée. Elle se situe, de fait, à l'intersection de deux axes historiques, en ce qu'elle participe tant de l'expansion des communications du début des années 1960, que du développement technologique des années 2000. Aussi, le cadre théorique conserve-t-il une pertinence contemporaine lorsqu'appliqué aux œuvres de Campbell.

D’entrée de jeu, il faut préciser que, pour la théorie de l'information, la notion de "signification " se distingue de celle d' "information " et qu'en outre, elle ne représente pas un véritable enjeu de la communication ${ }^{41}$, deux principes que soutiennent, avec quelques nuances, Eco et Campbell. En effet, pour Eco, l'information differe nettement de la signification, dans son entendement univoque habituel, pour se rapprocher 
plutôt de la "signification poétique " ou de la « richesse du sens esthétique d'un message "; pour Campbell, l'information est envisagée au sens mathématique, en nombre de bits, et s'oppose à la signification qui est comprise au sens poétique ${ }^{42}$. Ces différentes acceptions portent à confusion, mais elles ne modifient pas fondamentalement la présente argumentation sur l'expérience esthétique, car, comme il en sera question, tant pour Eco que pour Campbell, le « désordre » d'un message dessert la signification ordinaire au profit de la signification dite poétique. Cette dernière se définit, par ailleurs, de façon un peu vague comme une richesse interprétative attribuable à une ambiguïté organisée matériellement. Par souci de cohérence, le vocabulaire d'Eco, dans lequel l'information correspond à cette signification poétique, sera conservé et l'ouverture explicite des œuvres d'art, une ouverture due à une suggestion dirigée, sera comprise comme un accroissement d'information.

La théorie scientifique estime la mesure de l'information par une méthode probabiliste. Elle considère grosso modo qu'un message prévisible informera peu et, inversement, qu'un message imprévisible informera beaucoup. En un mot, elle associe l'information à la non-probabilité. C'est l'organisation d'un message qui en déterminerait la probabilité et sa désorganisation, la non-probabilité. La signification s'opposerait ainsi à l'information étant donné que, pour des raisons d'intelligibilité, elle se communique grâce à une organisation probable, par exemple, des conventions grammaticales ou syntaxiques, et qu'au contraire, l'information se transmet à travers une désorganisation improbable, originale, qui tend à enfreindre ces mêmes conventions. Dans ces circonstances, la quantité d'information s'avère directement proportionnelle à la désorganisation, ou plus exactement au désordre d'un message, du moins jusqu'à un certain seuil au-delà duquel le signal dégénère en bruit 43 . De là, Eco insiste sur le fait que les arts modernes, les œuvres ouvertes spécifiquement, utilisent le désordre, un certain type de désordre, pour augmenter l'information. Les œuvres littéraires, notamment, transgresseraient les normes de la langue, ses règles de construction, grâce à une désorganisation inattendue, ce qui maximiserait l'information communiquée, à savoir la signification poétique. Toutefois, le désordre issu d'une telle transgression ne s'assimilerait pas à un chaos absolu et, dans les faits, l'abandon d'un ordre établi n'éradiquerait pas toute forme d'organisation. Selon Eco, effectivement, les œuvres littéraires ouvertes briseraient les conventions du système linguistique, tout en conservant celui-ci, à défaut de quoi elles deviendraient incommunicables. Elles recréeraient ainsi, sur les bases du système en vigueur, un nouveau système possédant ses propres règles internes. Il existerait, par conséquent, un désordre souhaitable, organisé, qui accroît l'information, et un désordre non souhaitable, chaotique, qui la diminue, le premier étant lié au concept scientifique d'incertitude et le second, à celui de perturbation ou de bruit. En somme, l'œuvre ouverte serait un message organisé, dans lequel ont été sciemment intégrés des éléments de désordre qui rompent avec un ordre initial. Cette rupture d'un ordre initial, prévisible, tromperait les attentes et stimulerait les facultés imaginatives. Car, sous l'effet de l'incertitude, le lecteur serait poussé, dans sa démarche d'interprétation, à trouver par lui-même des réponses, des solutions satisfaisantes, et ce, en faisant appel à sa propre expérience qui, bien entendu, est indissociable d'un contexte socioculturel déterminé. Le désordre intentionnel correspondrait donc à une forme de suggestion dirigée, une suggestion qui sollicite un spectateur actif et qui, ce faisant, multiplie les possibilités interprétatives.

En appliquant le même raisonnement à la production de Jim Campbell, il appert que l'organisation inhabituelle des œuvres, leur haut degré d'ambiguïté visuelle, contribuerait à une incertitude qui maximise l'information. Par la remédiation des photographies nécrologiques en séquences vidéographiques à peine compréhensibles, l'artiste propose une configuration formelle inusitée qui rompt avec les normes établies du portrait, particulièrement avec la représentation de nature identitaire, et qui transgresse conséquemment un ordre prévisible. C'est ainsi que la très faible résolution, soit le peu d'information au sens mathématique, participerait d'un désordre qui contrecarre toute univocité et qui entraîne un accroissement d'information au sens poétique. Â ce stade, la logique argumentaire peut suivre une trajectoire qui calque implacablement le modèle scientifique et qui en arrive à chiffrer l'expérience esthétique. Eco évite cette aberration, que rejettera également Rudolf Arnheim, et s'en tient aux grands principes de communication de la théorie. Si la démarche de Campbell écarte, elle aussi, un tel réductionnisme, force est d'admettre cependant qu'elle s'intéresse de près à sa dynamique. L'artiste se risque, en effet, à évaluer en pixels un seuil dans l'expérience perceptuelle et à explorer les rapports, sous-entendus inversement proportionnels, entre signification poétique et information quantifiée (bits), d'où le titre de son projet, Explorations of Meaning in Quantized Information. Sur ce dernier point, il déclare : " $[\mathrm{An}]$ aspect of this project will be investigating what different types of low bit rate information might be most meaningful in the context of specific poetically oriented communications »44. Tout se passe comme si Campbell, l'artiste-ingénieur, réfléchissait sur les effets d'une confrontation entre objectivité mathématique et subjectivité humaine. Ainsi, le seuil de perception qu'il s'efforce de calculer, le seuil plus fictif que réel entre reconnaissance et non-reconnaissance, correspond théoriquement au point critique qui délimite incertitude et bruit : en diminuant la résolution de l'image jusqu'à la limite de la reconnaissance, Campbell chercherait à s'assurer d'un maximum d'incertitude, donc de signification poétique, tout en restant en deçà du seuil où le message se transformerait en bruit, c'est-à-dire en conservant un minimum d'ordre 
pour éviter la perte de communication. La résolution de 192 pixels correspondrait alors à la ligne de partage présumée entre un message ambigu, mais communicable, soit le portrait reconnaissable, et un message chaotique, donc incommunicable, soit le portrait non reconnaissable. Tout comme la résolution, les bruits aléatoires introduits dans la séquence vidéographique participent d'une organisation visuelle à la fois prédéterminée par l'artiste et imprévisible pour le spectateur. Ce brouillage sporadique des physionomies diffère d'une véritable perturbation qui ruinerait la communication : il s'agit plutôt de modules de désordre organisé qui simulent une difficulté de communication, sans en provoquer une, et qui procèdent de l'ouverture donnée par la suggestion dirigée. En dernière analyse, cette démonstration expose comment Campbell a pensé les stratégies visuelles afin de créer un impact chez le spectateur. Même si la quantification d'un seuil de reconnaissance s'avère un exercice illusoire, il convient de reconnaitre, à la décharge de l'artiste, que les œuvres possèdent l'ambiguïté suggestive propre au concept d'ouverture, et qu'en ce sens, il paraît légitime de concevoir qu'elles invitent à de libres associations imaginatives.

La profonde ambiguïté des portraits électroniques de Jim Campbell se révèle, sans nul doute, le principal déterminant de leur expérience perceptuelle et esthétique. L'imprécision produite par la configuration médiale, la reconnaissance incertaine des visages, nie la fonction traditionnelle du portrait, soit une fonction identitaire, et, par là, défie les attentes à l'égard de l'expérience du portrait. Privé de ses repères habituels, le spectateur s'efforcerait alors, presque à son insu, de lever l'incertitude, d'apporter une clarification, voire de compléter l'œuvre d'art en s'engageant activement dans le processus d'interprétation. Les portraits électroniques attiseraient donc l'imagination à la faveur d'un dispositif qui contrarie les habitudes perceptives. Ils infléchiraient la réponse spectatorielle dans des directions variées et contribueraient ainsi à décupler les possibilités de lecture. D'aucuns décèleraient alors dans la dissolution des visages tant le dysfonctionnement d'un processus cognitif que le caractère éphémère de la vie ou les limites fondamentales de la théorie de l'information. Quelle que soit la position interprétative choisie, il semble que chacune d'elles soit défendable, mais ce, jusqu'à un certain point, car, dans cet " accroissement d'information " de l'œuvre d'art, toute interprétation peut basculer dans la surinterprétation. Dans ces conditions, la signification des portraits, si tant est qu'une signification univoque existe, acquiert une valeur secondaire et tout effort d'analyse à son propos apparaît vite anecdotique. En fin de compte, Jim Campbell est le portraitiste improbable qui, en opacifiant à l'extrême les portraits d'Harry Nyquist et de Claude Shannon, les soustrait à une signification identitaire et, du même souffle, tend à les détacher de la tradition, tant historique que contemporaine, du portrait.
Notes

Cette recherche a été financée par le Conseil de recherches en sciences humaines.

1 Le projet a également reçu un soutien subsidiaire du Fleishhacker Foundation Eureka Award et du Rockefeller Foundation Multimedia Award.

2 B. S. génie électrique, 1978 ; B. S. mathématiques, 1978.

3 Richard Brilliant, Portraiture, $1^{\text {re }}$ éd. 1991, London, Reaktion Books, 2002, p. 31.

4 Leon D. Harmon, "The Recognition of Faces ", Scientific American, vol. CCXXIX, n ${ }^{\circ}$, 1973, p. 70-82.

5 Umberto Eco, "Ouverture, information, communication ", dans L'auvre ouverte, trad. Chantal Roux de Bézieux, $1^{\text {re }}$ éd. 1962, Paris, Seuil, 1965, p. 67-114.

6 Le « bruit visuel » ou " bruit optique » est une perturbation qui altère un signal optique.

7 Harry Nyquist, "Certain Topics in Telegraph Transmission Theory", Transactions of the American Institute of Electrical Engineers, vol. XLVII, n 2, avril 1928, p. 617-44 ; Claude E. Shannon, «A Mathematical Theory of Communication ", The Bell System Technical Journal, vol. XXVII, juillet/octobre 1948, p. 379-423, 623-56.

8 Le terme bit vient de binary digit. Il s'agit d'une unité d'information popularisée par les travaux de Claude Shannon.

9 La notion de remédiation est employée dans le sens que lui confèrent les auteurs Jay David Bolter et Richard Grusin : " the formal logic by which new media refashion prior media forms ». Jay David Bolter et Richard Grusin, Remediation : Understanding New Media, Cambridge, MA, MIT Press, 1999, p. 273.

10 Jim Campbell, « Proposal to The Daniel Langlois Foundation for Art, Science, and Technology : Submitted for a Research Grant for Individual Artists ", Collection Fondation Daniel Langlois, Cinémathèque québécoise, Montréal, 28 janvier 2000, s. p.

11 La théorie de l'information n'est pas sans participer à l'émergence des sciences cognitives. L'année 1956 est en effet marquante dans la constitution d'un mouvement intellectuel qui prendra plus tard le nom de «sciences cognitives ». Elle correspond à la tenue au MIT d'un Symposium on Information Theory.

12 Mark Rowlands, The New Science of the Mind: From Extended Mind to Embodied Phenomenology, Cambridge, MA, MIT Press, 2010, p. 111

13 Voir Richard C. Atkinson et Richard M. Shiffrin, «Human Memory: A Proposed System and its Control Processes ", dans K. Spence et J. Spence (dir.), The Psychology of Learning and Motivation, New York, Academic Press, 1968, p. 89-195; Philip Johnson-Laird, The Computer and the Mind: An Introduction to Cognitive Science, Cambridge, MA, Harvard University Press, 1988.

14 Pierre Steiner, "Introduction : cognitivisme et sciences cognitives ", Labyrinthe, vol. XX, n 1, 2005, p. 13. 
15 Voir Claudette Fortin et Robert Rousseau, Psychologie cognitive : une approche de traitement de l'information, Sillery, Presses de l'Université du Québec, 1989.

16 Il existe deux façons d'envisager le parallèle entre humain et ordinateur : le cerveau peut être comparé à l'objet-ordinateur (hardware) et l'esprit humain au programme informatique qui permet à la machine de traiter l'information (software). L'analogie est ici limitée à celle entre esprit et programme. Au sujet de cette analogie, voici ce qu'énonce Georges Thinès : "Indépendamment du point de vue biologique, la psychologie cognitive tente depuis le début des années soixante-dix de renouveler la théorie de la perception en proposant des modes d'analyse inspirés par la théorie de l'information et de la communication. Cette tendance de la psychologie expérimentale se fonde essentiellement sur l'idée que la perception est un acte cognitif, en ce sens que le phénomène que l'on désigne de cette façon est le résultat d'un traitement subjectif guidé par un programme, celui-ci orientant les stratégies du comportement. Le terme de "cognition" couvre donc tous les processus par lesquels l'information qui atteint le sujet est transformée, élaborée, mise en mémoire et finalement utilisée ". Georges Thinès, "Perception ", dans Encyclopaedia Universalis, http://www.universalis-edu.com (consulté le 15 août 2013).

17 Un algorithme est un ensemble de règles ou d'instructions servant à la résolution d'un problème, tel que l'exécution d'un calcul, en un nombre fini d'étapes ; le programme informatique est l'intégration de cet algorithme dans un langage de programmation.

18 Claude E. Shannon, "Communication in the Presence of Noise", Proceedings of the Institute of Radio Engineers, vol. XXXVII, $\mathrm{n}^{\circ} 1$, janvier 1949, p. 10-21.

19 Aaron D. Wyner et Shlomo Shamai, "Introduction to "Communication in the Presence of Noise" by C. E. Shannon ", Proceedings of the IEEE, vol. LXXXVI, n² 2, février 1998, p. 442-46.

20 Avant cette expérimentation, Leon Harmon et son collègue, Kenneth Knowlton, avaient créé une image de grand format, un nu intitulé Studies in Perception I, qui constitue l'un des premiers exemples de traitement d'image numérique. L'image était réalisée à partir d'une photographie analogique qui avait été numérisée à l'aide d'un appareil particulier et convertie en une multitude de symboles typographiques. La forme humaine n'était perceptible qu'à partir d'une certaine distance, tandis que, de près, le grain de la typographie créait un effet " pointilliste » dissolvant l'image jusqu'à l'abstraction.

21 Steve Dietz, "The Difference that Makes a Difference : The Liminal Art of Jim Campbell ", dans Sarah King (dir.), Quantizing Effects : The Liminal Art of Jim Campbell, Santa Fe, Site Santa Fe, 2005, p. 16 ; Campbell, "Proposal ", s. p. ; le site web de l'artiste expose aussi une image réalisée lors de l'expérimentation de Harmon, mais sans y joindre de texte explicatif. Jim Campbell, http://www.jimcampbell.tv/ (consulté le 24 juillet 2013).

22 La manipulation est effectuée à partir d'un billet de cinq dollars américain, qui lui-même reproduit une photographie de 1864 prise par Mathew Brady (1823-1896).

23 Harmon, op. cit., p. 70-82.

24 Harmon formule la question suivante : " To what extent is recognition impaired when the image of a face is blurred or otherwise degraded ?". Harmon, ibid., p. 71.

25 Harmon, ibid., p. 72.

26 Richard Gregory, Seeing Through Illusions, Oxford et New York, Oxford University Press, 2009, p. 116-17.

27 Campbell cité dans Meg Grey, "Jim Campbell », RES : The Future of Filmmaking, vol. V, n 3, 2002, p. 79.

28 Le format des portraits électroniques est adapté à la forme d'un buste et est, par conséquent, plus étroit que celui des images scientifiques : $16 \times 12$ pixels au lieu de $16 \times 16$ pixels. Les 64 pixels excédentaires mesurés par Harmon correspondent ainsi à l'espace vide du fond sur les côtés du visage, ce qui signifierait que le seuil quantifié de reconnaissance faciale obtenu par l'artiste et le scientifique est, en fait, exactement le même. Notons également que la résolution du portrait de Lincoln n'est pas celle qui déterminera le résultat final de l'expérimentation de Harmon.

29 Harmon, op. cit., p. 76.

30 Les premiers essais techniques tentés par l'artiste anticipent la démarche. Ils sont brièvement présentés dans la description du projet pour la Fondation Daniel Langlois : " [I]f one looks at an image from far away that has extremely large pixels (the first one that I am aware of was done by Leon Harmon at Bell Labs using an image of Abraham Lincoln in 1973) the pixels are not seen or perceived. The viewer can only see the "image". [...] I have been doing some very simple experiments with large pixelated images. It is interesting to note that putting a crude filter, like a piece of sand blasted [sic] glass, between the viewer and the image causes the image to be more comprehensible ». Campbell, "Proposal », s. p.

31 Dietz, ibid., p. 16.

32 Un aperçu représentatif, mais non exhaustif : Umberto Eco, ibid., p. 67-114; Abraham Moles, Théorie de l'information et perception esthétique, Paris, Flammarion, 1958 ; les articles réunis sous la direction de Rudolf Arnheim dans The Journal of Aesthetics and Art Criticism, vol. XVII, n 4, 1959, p. 501-22.

33 Ce colloque, sous la présidence de Rudolf Arnheim et intitulé Information Theory and the Arts, a lieu en 1957 lors du congrès de l'Association américaine de psychologie à New York.

34 Rudolf Arnheim, Entropy and Art: An Essay on Disorder and Order, Berkeley, University of California Press, 1971, p. 18.

35 Eco, ibid., p. 67-114. Voir aussi : Dale D. Drum, "Change, Meaning, and Information ", Journal of Communication, vol. VII, $\mathrm{n}^{\circ}$ 4, 1957, p. 161-70 ; Leonard B. Meyer, "Meaning in Music and Information Theory ", The Journal of Aesthetics and Art Criticism, vol. XV, $\mathrm{n}^{\circ}$ 4, juin 1957, p. 412-24.

36 Umberto Eco, L'œuvre ouverte, trad. Chantal Roux de Bézieux,

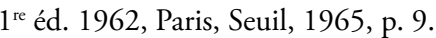


37 Eco, ibid., p. 51.

38 Eco, ibid., p. 54.

39 Jim Campbell, "Final Project Report for Exploration of Meaning in Quantized Information ", Collection Fondation Daniel Langlois, Cinémathèque québécoise, Montréal, 18 août 2001, s. p.

40 Eco, op. cit., p. 53.

41 Dans l'introduction explicative à la deuxième publication de la théorie de l'information de Shannon, Warren Weaver insiste : « information must not be confused with meaning ». Claude Shannon et Warren Weaver, The Mathematical Theory of Communication, $1^{\mathrm{re}}$ éd. 1948, Urbana, University of Illinois Press, 1964, p. 8.

42 L'artiste écrit : "Information refers specifically to information in the mathematical sense, while the concept of meaning embodies the meaning in the poetic sense. The oxymoron title of this series, "Ambiguous Icons", indicates the fundamental difference between information and meaning, namely that poetic meaning is ambiguous (or plural) and mathematical information is precise (or singular) ». Campbell, «Final Project Report », s. p.

43 Afin de calculer l'information, la théorie emprunte un concept issu de la thermodynamique, à savoir l'entropie, qui tient lieu, dans son adaptation pour les communications, de mesure statistique du degré de désordre d'un message.

44 Campbell, « Proposal ", s. p. 